\title{
Hemostasia hepática utilizando eletrocautério seco ou emplastrado com lidocaína ou neomicina ou glicerina ou vaselina, em coelho
}

\section{Liver hemostasis using a dry eletrocautery or greased with lidocaine or neomycin or glycerin or vaseline, in rabbit}

\author{
Argos Soares de Matos Filho"; Andy Petroianu, TCbC-MG²; Luiz Ronaldo Alberti, ACbC-MG³; Paula Vieira Teixeira Vidigal ${ }^{4}$; \\ Daniel Cruz Ferreira dos Reis ${ }^{5}$; Davi Machado de Souza ${ }^{5}$
}

\section{R E S U M O}

\begin{abstract}
Objetivo: Avaliar a hemostasia e a cicatrização hepática após hepatectomia segmentar, utilizando eletrocautério seco, ou emplastrado com: gel de lidocaína, pomada de neomicina, loção de glicerina e pomada de vaselina. Métodos: Coelhos foram submetidos à hepatectomia parcial e distribuídos em seis grupos $(n=10)$ : Grupo 1: sem tratamento; Grupo 2: tratamento com eletrocautério seco; Grupo 3: emplastrado com gel de lidocaína; Grupo 4: pomada de neomicina; Grupo 5: loção de glicerina; Grupo 6: pomada de vaselina. Foram mensurados o peso do fígado ressecado, o volume de sangramento e o tempo dispendido para hemostasia. Cinco coelhos de cada grupo foram reoperados após 24 horas, e cinco após sete dias, para biópsia da ferida hepática e exploração da cavidade abdominal. Eritrograma e marcadores de função e lesão hepática foram avaliados no pré-operatório e antes das reoperações. Resultado: O gel de lidocaína e a loção de glicerina reduziram o volume do sangramento e o tempo de hemostasia, além de conduzirem a energia térmica do eletrocautério, provocando degeneração hidrópica celular após 24 horas e necrose após sete dias, com profundidade maior no tecido hepático. Todas as substâncias elevaram as aminotransferases. Esses valores normalizaram-se em até sete dias. Conclusão: O eletrocautério emplastrado com gel de lidocaína e a loção de glicerina foram os métodos mais eficazes na hemostasia do parênquima hepático de coelhos.
\end{abstract}

Descritores: Hepatectomia. Hemostasia cirúrgica. Eletrocoagulação. Lidocaína. Glicerina.

\section{INTRODUÇÃO}

\begin{abstract}
A hemorragia hepática após trauma acidental ou durante procedimentos operatórios pode ser intensa e de difícil controle. Cabe ao cirurgião a hemostasia adequada durante ressecções ou lesões maiores do parênquima hepático, para prevenir complicações e óbitos. O volume de sangue perdido é decisivo no prognóstico pós-operatório'

O extravasamento de sangue durante operações hepáticas não é incomum, mesmo quando o sangramento não é de difícil controle ${ }^{2}$. A predisposição desse órgão para hemorragia difusa está relacionada à sua vascularização e estrutura sinusoidal, sem musculatura lisa capaz de promover vasoconstrição.

Apesar de reconhecer-se a necessidade de disseç̧ão do parênquima hepático com sangramento mínimo, em muitos casos, impõe-se a transfusão de elementos sanguíneos, com risco de transmissão de doenças virais, reação de hipersensibilidade e imunodepressão $0^{3,4}$. A hemorragia intensa acompanhada de múltiplas transfusões
\end{abstract}

está relacionada à menor sobrevida, devido ao seu efeito sistêmico, com consequente insuficiência de múltiplos órgãos ${ }^{5}$.

Durante ressecção hepática convencional, apesar do controle do tronco da artéria hepática e da veia porta para o segmento a ser ressecado, o sangramento ainda ocorre, por retorno venoso. Vários cirurgiões usam manobras de exclusão vascular total ou seletiva, na tentativa de diminuir a perda sanguínea. Entretanto, essa conduta acompanha-se de transtornos hemodinâmicos e metabólicos, com disfunção hepática pós-operatória, mal tolerada, principalmente por pacientes hepatopatas crônicos, que possuem baixa reserva funcional ${ }^{6}$.

Ressecções hepáticas são indicadas para tratar neoplasias primárias ou metastáticas do fígado. Embora a mortalidade desses procedimentos tenha diminuído, sua morbidade permanece alta, principalmente em decorrência do tempo operatório e da perda sanguínea, acentuando-se nos pacientes com icterícia e cirrose. A ressecção hepática em cirróticos é mais grave, devido ao risco maior

Trabalho realizado no Departamento de Cirurgia da Faculdade de Medicina da Universidade Federal de Minas Gerais - UFMG, Belo Horizonte, MG, Brasil.

1. Mestre e Doutorando em Cirurgia da UFMG-MG-BR. 2. Professor Titular do Departamento de Cirurgia da Faculdade de Medicina da UFMGMG-BR. 3. Professor Adjunto do Departamento de Cirurgia da Faculdade de Medicina da UFMG-MG-BR. 4. Professora Adjunta do Departamento de Anatomia Patológica da Faculdade de Medicina da UFMG-MG-BR. 5. Acadêmico de Medicina da UFMG-MG-BR. 
de sangramento, reserva hepática limitada, carência de propriedades regenerativas do parênquima e predisposição dos pacientes a sepse ${ }^{7,8}$. Pacientes cirróticos sangram mais também por causa de hipertensão porta, associada a coagulopatias.

Adquirir e manter hemostasia depende de vários fatores, incluindo técnicas cirúrgicas e anestésicas. A manutenção de uma pressão venosa central baixa pode auxiliar na operação, mas necessita de avaliação pré-operatória, para verificar se há condições cardíacas adequadas para esse procedimento. O pinçamento de Pringle do pedículo hepático permite considerável redução do sangramento, embora seja ineficaz na hemorragia proveniente da veia hepática e de seus afluentes ${ }^{9}$.

A maior perda sanguínea na ressecção hepática decorre da secção de pequenos vasos, que não são previamente ligados. Para evitar a hemorragia, que pode ser acompanhada de bilerragia, é fundamental obter-se superfície de corte seca antes do fechamento do abdome.

As técnicas para dissecção do parênquima hepático têm mudado da digitoclasia e uso do eletrocautério para diversos dispositivos de secção hepática, como o aspirador ultrassônico, o dissecador de jato d'água, ablação por radiofrequência, coagulação por microondas e bisturi de argônio, entre outros. Esses métodos reduzem o sangramento, embora nenhum deles seja o ideal. A técnica de digitoclasia ${ }^{10}$ e de dissecção por sucção ${ }^{11}$ permitem o isolamento e ligadura vascular e biliar, mas não controlam os pequenos vasos parenquimatosos, requerendo que o cirurgião lance mão de outras alternativas cirúrgicas.

Originalmente utilizadas em pacientes com contra-indicação para operações, as técnicas de ablação térmica, como o LASER, microondas ou radiofrequência, tornaram-se alternativa útil para o tratamento de tumores hepáticos. Todas têm como princípio a produção de energia térmica, levando a necrose por coagulação nos teci$\operatorname{dos}^{12}$. A radiofrequência é a técnica de ablação térmica mais utilizada em todo o mundo, devido à segurança e bons resultados. Uma limitação associada a esse método é a necessidade de múltiplas ablações sequenciais. Os grampeadores tornaram-se muito utilizados na prática operatória e são úteis para seccionar e ocluir vasos do parênquima hepático.

Agentes tópicos têm sido propostos como medida adjuvante para facilitar a hemostasia de órgãos parenquimatosos. Entre essas substâncias, destacam-se o colágeno microfibrilar e os selantes de fibrina. Alguns compostos, como a cola formaldeído-resorcina-gelatina (GRF) e adesivos de cianoacrilato não se tornaram agentes hemostáticos tópicos de rotina, devido à dificuldade de aderência a superfícies úmidas e também por causa de sua histotoxidade.

Em 2004, durante hepatectomia segmentar para tratar carcinoma hepático, Petroianu utilizou o eletrocautério emplastrado com gel de lidocaína, por ser esse o único gel disponível naquela oportunidade na sala operatória. Sob a ação do calor do eletrocautério, o gel aderiu sobre a superfície cruenta do fígado, formando uma crosta, que inter- rompeu a hemorragia dos vasos menores. Após o sucesso obtido nessa ocasião, o método passou a ser utilizado tanto em operações no fígado, quanto baço, em esplenectomias parciais, e, mais recentemente, secções ósseas. A facilidade e eficácia desse procedimento permitiu sua utilização em operações laparoscópicas para biópsia esplênica e esplenectomias subtotais ${ }^{13,14}$.

O objetivo deste trabalho foi avaliar a hemostasia e a cicatrização do parênquima hepático após hepatectomia segmentar, utilizando eletrocautério seco ou emplastrado com gel de lidocaína, pomada de neomicina, loção de glicerina ou pomada de vaselina.

\section{MÉTODOS}

Este estudo foi aprovado pela Câmara do Departamento de Cirurgia da Faculdade de Medicina da Universidade Federal de Minas Gerais (UFMG) e pelo Comitê de Ética em Experimentação Animal da UFMG (CETEA).

Foram pesquisados 60 coelhos Nova Zelândia albinos (Oryctolagus cuniculus) adultos do sexo masculino, alocados em gaiolas separadas, onde receberam alimentação apropriada além de água à vontade.

A distribuição dos animais foi feita aleatoriamente em seis grupos $(n=10)$ :

controle)

Grupo 1: sem hemostasia hepática (grupo

eletrocautério seco

\section{Grupo 2: hemostasia hepática com}

Grupo 3: hemostasia hepática com eletrocautério emplastrado com gel de lidocaína.

Grupo 4: hemostasia hepática com eletrocautério emplastrado com pomada de neomicina.

Grupo 5: hemostasia hepática com eletrocautério emplastrado com loção de glicerina.

Grupo 6: hemostasia hepática com eletrocautério emplastrado com pomada de vaselina.

Amostra de sangue foi coletada no pré-operatório em todos os animais, por meio de punção da artéria central da orelha direita com cateter $n^{\circ} 24$, após dilatação da artéria com gaze embebida com xilol sobre a orelha do animal, para realização de eritrograma e dosagem de plaquetas, dosagem da razão normatizada internacional (RNI), tempo de protrombina e tempo de tromboplastina parcial e dosagem de fosfatase alcalina, gama-glutamil transpeptidase, aspartato aminotransferase e alanina aminotransferase.

Os animais foram anestesiados com injeção de cloridrato de quetamina $(80 \mathrm{mg} / \mathrm{kg}$ ) associado com cloridrato de xilazina (15 mg/kg) intramuscular na região glútea e mantidos em respiração espontânea. Durante todo o período de anestesia, foram observadas as frequências cardíaca e respiratória, além da movimentação voluntária dos coeIhos, com vista a detectar o nível da anestesia e possíveis complicações anestésicas. Não foram necessárias doses suplementares de anestésico. 
Após a indução anestésica, os coelhos foram colocados em decúbito dorsal sobre uma mesa operatória para animais de médio porte, mantendo suas patas abduzidas. Em condições de anti-sepsia, os coelhos foram submetidos à laparotomia mediana supra-umbilical de sete centímetros de comprimento a partir do apêndice xifóide, no sentido crânio-caudal. Explorou-se a cavidade abdominal, visando a identificar alterações que poderiam excluir o animal deste trabalho.

Em seguida, um segmento do lobo hepático esquerdo foi exteriorizado através da laparotomia e seccionado pelo cirurgião com tesoura curva, enquanto o auxiliar o mantinha seguro com uma gaze. Esse segmento teve as mesmas dimensões em todos os animais. $\quad \mathrm{N}$ o Grupo 1 (controle) nenhum tratamento foi realizado na ferida hepática. No Grupo 2 foi realizada hemostasia, com aplicação do eletrocautério a 110V, $30 \mathrm{~W}$, seco sobre a superfície da ferida. Nos grupos seguintes a hemostasia foi realizada com o eletrocautério, com a mesma intensidade elétrica, emplastrado com gel de lidocaína (Grupo 3), pomada de neomicina (Grupo 4), loção de glicerina (Grupo 5) e pomada de vaselina (Grupo 6).

Em todos os grupos, o sangramento per-operatório foi quantificado, mediante o peso das gazes utilizadas durante a hemostasia. Quantificou-se o tempo operatório, e verificou-se a interrupção completa do sangramento. Após a revisão da cavidade, a parede abdominal foi fechada em dois planos.

Decorridas 24 horas pós-operatórias, foram coIhidas novas amostras de sangue em todos os animais, para dosagem de eritrócitos, hematócrito, hemoglobina, alanina aminotransferase, aspartato aminotransferase, gamaglutamil transpeptidase e fosfatase alcalina. Em cada um dos seis grupos, cinco coelhos foram reoperados. Os outros cinco animais foram reoperados após sete dias, quando foi realizada nova coleta de sangue para dosagem da alanina aminotransferase, aspartato aminotransferase, gamaglutamil transpeptidase e fosfatase alcalina. Após anestesia com cloridrato de quetamina $(80 \mathrm{mg} / \mathrm{kg}$ ) por via intramuscular e reabertura da parede abdominal, foi realizada a exploração da cavidade dos coelhos, com vista a detectar aderências ou coleções, sendo retirado fragmento do fígado, envolvendo a ferida hepática e parte do parênquima aparentemente íntegro. Em seguida, a morte dos animais foi induzida com injeção de $10 \mathrm{~mL}$ de cloreto de potássio $10 \%$ na veia cava caudal. Em todos os grupos, o material coletado foi submetido à análise histológica, após a rotina de preparação anatomopatológica e coloração pela hematoxilina e eosina.

Os dados foram apresentados como média \pm erro padrão da média. Todos os resultados foram inicialmente avaliados pelo teste de normalidade de Kolmogorov-Smirnov. Os dados apresentaram distribuição normal e mesma variância. Para comparação do peso do fragmento hepático retirado, da quantidade de sangramento e do tempo para adquirir hemostasia foi realizado o teste paramétrico de análise de variância (ANOVA) seguido pelo teste de comparação múltipla de Tukey-Kramer. Para comparação dos valores hematimétricos e bioquímicos foi realizado o teste de análise de variância (ANOVA) para amostras pareadas seguido pelo teste de comparação múltipla de Tukey-Kramer. Todos os resultados foram considerados significativos para valores comparativos inferiores a 5\% ( $p<$ 0,05).

\section{RESULTADOS}

Todos os coelhos toleraram bem a anestesia e evoluíram satisfatoriamente, com recuperação pósanestésica rápida e atividade motora aparentemente normal dentro das gaiolas (Tabela 1).

Tabela 1 - Média do peso do sangue perdido, tempo de sangramento, e achados na reoperação dos coelhos por grupo.

\begin{tabular}{|c|c|c|c|}
\hline Grupo & $\begin{array}{l}\text { Peso do } \\
\text { sangue } \\
\text { perdido }(g)\end{array}$ & $\begin{array}{l}\text { Tempo de } \\
\text { sangramento } \\
\text { (segundos) }\end{array}$ & Achados na reoperação* \\
\hline 1 & 9,33 & 569,10 & $\begin{array}{l}24 \text { horas: Coágulo sobre a ferida hepática ( } 1 \text { animal). } \\
7 \text { dias: Aderência do omento à ferida em ( } 2 \text { animais). }\end{array}$ \\
\hline 2 & 4,96 & 205,10 & $\begin{array}{l}24 \text { horas: Aderência de lobo adjacente à ferida hepática ( } 2 \text { aimais). } \\
7 \text { dias: Aderência do omento ( } 1 \text { animal) e lobo adjacente ( } 1 \text { animal). }\end{array}$ \\
\hline 3 & 3,19 & 278,80 & $\begin{array}{l}24 \text { horas: Aderência do omento à ferida hepática ( } 2 \text { aimais). } \\
7 \text { dias: Aderência de lobo adjacente à ferida hepática ( } 5 \text { aimais). }\end{array}$ \\
\hline 4 & 4,18 & 330,90 & $\begin{array}{l}7 \text { dias: Aderência de lobo adjacente ( } 5 \text { animais) e do omento ( } 1 \text { animal) } \\
\text { à ferida hepática. }\end{array}$ \\
\hline 5 & 2,61 & 149,20 & $\begin{array}{l}7 \text { dias: Aderência de lobo adjacente ( } 4 \text { animais) e do omento ( } 1 \text { animal) } \\
\text { à ferida hepática. }\end{array}$ \\
\hline 6 & 10,04 & 523,6 & $\begin{array}{l}24 \text { horas: Aderência do omento à ferida em ( } 2 \text { animais). } \\
7 \text { dias: Aderência do omento ( } 5 \text { animais), e lobo adjacente, estômago e } \\
\text { parede abdominal ( } 1 \text { animal) à ferida hepática. }\end{array}$ \\
\hline
\end{tabular}

\footnotetext{
* Coelhos 1 a 5 reoperados após 24 horas; coelhos 6 a 10 reoperados após 7 dias.
}

1 = sem tratamento (controle); 2 = Tratamento com eletrocautério seco; 3 = Tratamento com eletrocautério emplastrado com lidocaína; $4=$ Tratamento com eletrocautério emplastrado com neomicina; 5 = Tratamento com eletrocautério emplastrado com glicerina; 6 = Tratamento com eletrocautério emplastrado com vaselina. 
No Grupo 1, a hepatectomia sem tratamento foi realizada sem complicações intra-operatórias nos 10 animais. No período de 10 minutos, quando foi realizada laparorrafia, apenas em três, o sangramento cessou.

No Grupo 2, um coelho apresentou sangramento copioso de difícil controle durante a tentativa de hemostasia, devido à secção de um vaso maior. Nos outros nove a hepatectomia parcial com hemostasia utilizando eletrocautério seco foi realizada sem complicações intraoperatórias.

O tempo de sangramento dos grupos que utilizaram eletrocautério seco e emplastrado com gel de lidocaína e pomada de neomicina foram menores que o grupo sem tratamento e que utilizou eletrocautério emplastrado com pomada de vaselina. O tempo de sangramento do grupo que utilizou eletrocautério emplastrado com glicerina foi ainda inferior ao que utilizou pomada de neomicina (Figura 1-A).

O volume de sangramento dos grupos que utilizaram eletrocautério seco ou emplastrado com gel de lidocaína, pomada de neomicina ou loção de glicerina foram equivalentes. Apenas os grupos que utilizaram gel de lidocaína ou loção de glicerina foram menores que do grupo sem tratamento ou que utilizaram pomada de vaselina (Figura 1-B).

O gel de lidocaína (Figura 2 A e B) conduziu bem a corrente elétrica através do tecido hepático, atingindo níveis mais profundos que o grupo que utilizou o eletrocautério seco, entre 0,8 e 1,5 cm. Cerca de 24 horas após as operações, a borda da ferida hepática apresentava uma crosta de consistência firme e esbranquiçada, não sendo possível distinguir qualquer estrutura vascular ou biliar. Essa crosta ocluiu os vasos sanguíneos e biliares, impedindo sangramento ou vazamento biliar. No entanto, o tempo para hemostasia nesse grupo foi superior ao grupo que utilizou o eletrocautério seco, em decorrência do período gasto para o gel solidificar sobre a superfície hepática.

Após cerca de 24 horas, as células hepáticas apresentavam sinais de "sofrimento", com coloração acidófila, indicando degeneração hidrópica dos hepatócitos. Entretanto, não se pode ainda falar em morte celular. Ela pôde ser observada após as biópsias realizadas no $7^{\circ}$ dia pós-operatório quando se observou picnose e cariólise. A regeneração estava presente nesses cortes pela evidência de tecido de granulação e neoformação de vasos e ductos biliares. Essas alterações atingiram maior profundidade tecidual nos grupos em que se utilizou o eletrocautério emplastrado com lidocaína e com glicerina. No grupo sem tratamento e em que se utilizou a pomada de vaselina, essas alterações foram menores, com formação de coágulo sobre a ferida hepática (Tabela 2).

\section{DISCUSSÃO}

Foram utilizadas substâncias tópicas disponíveis comercialmente. Idealmente, para hemostasia elas devem ser efetivas e seguras, sem efeito adverso associado com sua aplicação ou seus produtos gerados pelo calor. Essas substâncias devem ser capazes de formar coágulo e auxiliar na cicatrização da ferida, serem de fácil aplicação e baixo custo ${ }^{3}$.

A pomada de neomicina, não reduziu o sangramento quando se comparou com o eletrocautério seco, o que pode ser explicado pela ausência de conteúdo aquoso na composição das pomadas.
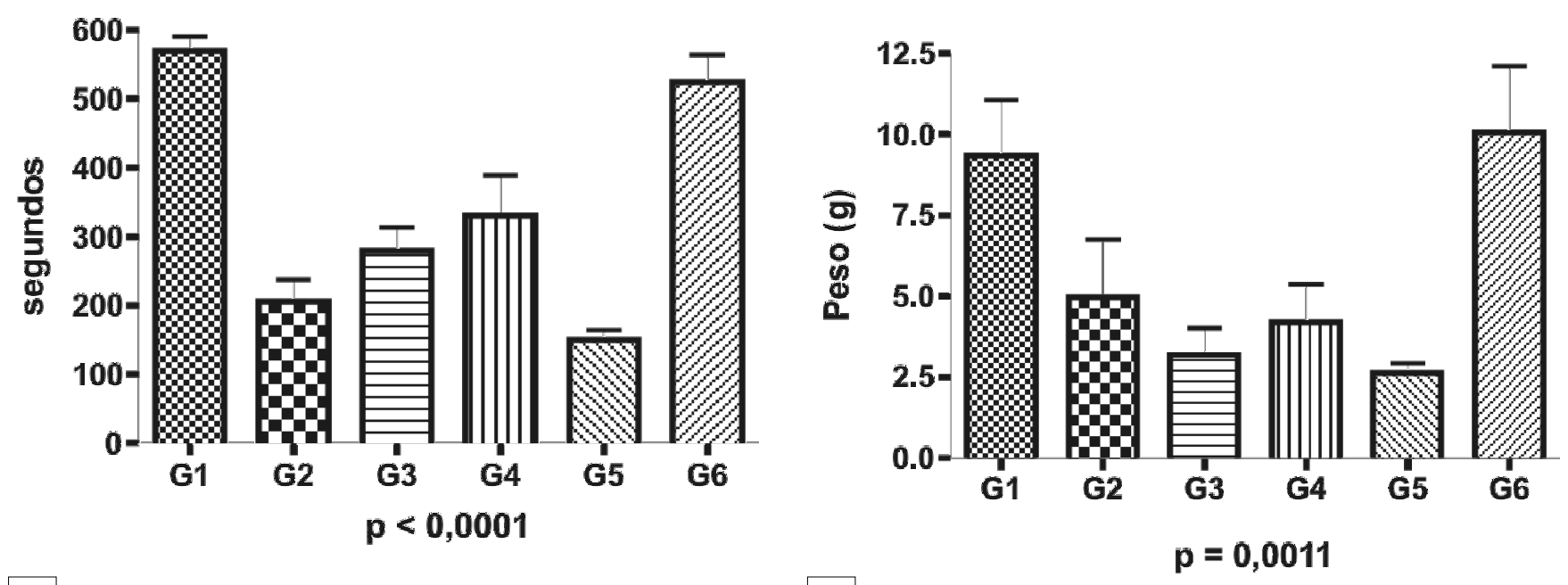

$$
\begin{gathered}
\mathrm{G} 2=\mathrm{G} 3=\mathrm{G} 4<\mathrm{G} 1=\mathrm{G} 6 \\
\mathrm{G} 5<\mathrm{G} 4
\end{gathered}
$$

$$
\mathbf{G} 2=\mathrm{G} 3=\mathbf{G} 4=\mathbf{G} 5 \quad \mathrm{G} 3=\mathbf{G} 5<\mathrm{G} 1=\mathrm{G} 6
$$

Figura 1 - Comparação do tempo de sangramento (A) e volume de sangramento (B) entre os grupos (ANOVA e comparação múltipla de Tukey-Kramer).

G1 = Sem tratamento (controle); G2 = Tratamento com eletrocautério seco; G3 = Tratamento com eletrocautério emplastrado com lidocaína; G4 = Tratamento com eletrocautério emplastrado com neomicina; G5 = Tratamento com eletrocautério emplastrado com glicerina; $\mathrm{G6}$ = Tratamento com eletrocautério emplastrado com vaselina. 

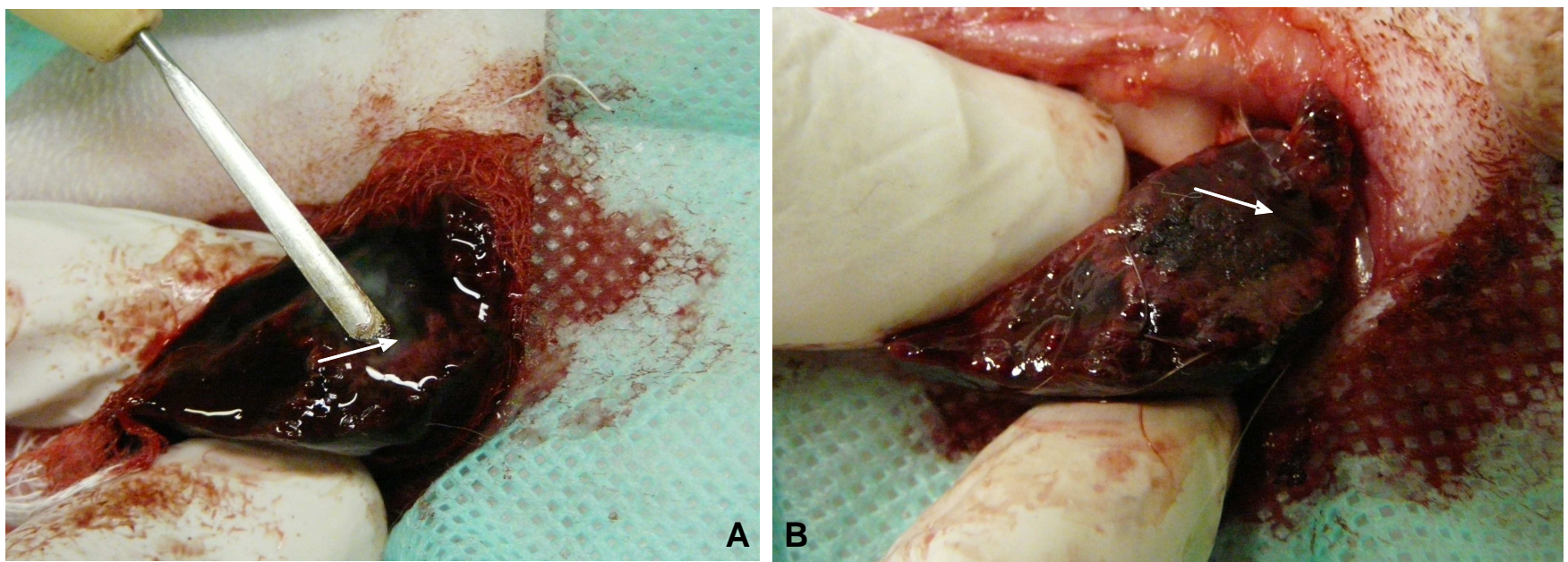

Figura 2

Hemostasia da ferida hepática utilizando eletrocautério emplastrado com gel de lidocaína. A - Eletrocautério emplastrado com lidocaína hemostasiando área cruenta do parênquima hepático (seta). B - Superfície hepática hemostasiada (seta).

Tabela 2 - Resultados dos exames laboratoriais dos animais submetidos à hepatectomia parcial.

\begin{tabular}{|c|c|c|c|c|c|c|c|}
\hline \multirow[b]{2}{*}{ Grupo } & \multicolumn{7}{|c|}{ Exame } \\
\hline & $\begin{array}{l}\text { Hemoglobina } \\
\text { (g/dl) }\end{array}$ & $\begin{array}{l}\text { Hemácias } \\
\text { (Hem/dl) }\end{array}$ & $\begin{array}{c}\text { Hematócrito } \\
(\%)\end{array}$ & $\begin{array}{l}\text { AST } \\
(u / I)\end{array}$ & $\begin{array}{l}\text { ALT } \\
(u / I)\end{array}$ & $\begin{array}{c}\text { FA } \\
(u / I)\end{array}$ & $\begin{array}{l}\text { G G T } \\
(u / I)\end{array}$ \\
\hline 1 Pré-oper. & $13,3 \pm 0,8$ & $6,5 \pm 0,5$ & $42,6 \pm 1,9$ & $66,1 \pm 26,8$ & $64,1 \pm 45,2$ & $151,1 \pm 51,1$ & $9,0 \pm 3,3$ \\
\hline 24 horas & $12,1 \pm 1,6$ & $5,8 \pm 0,6$ & $39,4 \pm 4,6$ & $239,6 \pm 170,6$ & $210,7 \pm 104,5$ & $106,8 \pm 40,4$ & $10,0 \pm 4,1$ \\
\hline 7 dias & - & - & - & $123,2 \pm 152,3$ & $58,6 \pm 25,4$ & $39,8 \pm 14,9$ & $11,0 \pm 3,5$ \\
\hline 2 Pré-oper. & $13,0 \pm 1,3$ & $6,3 \pm 0,5$ & $41,9 \pm 4,0$ & $72,9 \pm 44,8$ & $60,6 \pm 28,5$ & $156,5 \pm 35,9$ & $7,9 \pm 1,7$ \\
\hline 24 horas & $12,5 \pm 1,1$ & $6,1 \pm 0,6$ & $40,5 \pm 3,4$ & $266,3 \pm 64,7$ & $214,6 \pm 80,6$ & $137,4 \pm 64,6$ & $11,2 \pm 6,4$ \\
\hline 7 dias & - & - & - & $49,2 \pm 27,6$ & $48,0 \pm \quad 5,8$ & $36,8 \pm 13,3$ & $10,8 \pm 3,6$ \\
\hline 3 Pré-oper. & $13,6 \pm 1,2$ & $6,5 \pm 0,5$ & $43,1 \pm 3,3$ & $52,8 \pm 17,9$ & $75,2 \pm 29,9$ & $164,1 \pm 44,2$ & $6,8 \pm 1,5$ \\
\hline 24 horas & $12,2 \pm 0,8$ & $5,9 \pm 0,5$ & $39,4 \pm 2,3$ & $391,3 \pm 124,7$ & $393,9 \pm 119,6$ & $134,6 \pm 34,3$ & $7,1 \pm 2,5$ \\
\hline 7 dias & - & - & - & $132,8 \pm 173,8$ & $25,2 \pm 15,31$ & $62,4 \pm 24,3$ & $18,6 \pm 16,6$ \\
\hline 4 Pré-oper. & $12,7 \pm 1,1$ & $6,3 \pm 0,5$ & $41,3 \pm 3,2$ & $47,4 \pm 19,8$ & $39,2 \pm 18,5$ & $140,4 \pm 34,3$ & $7,3 \pm 1,1$ \\
\hline 24 horas & $11,6 \pm 0,7$ & $5,8 \pm 0,6$ & $38,2 \pm 3,3$ & $286,3 \pm 155,3$ & $147,2 \pm 59,0$ & $122,3 \pm 19,8$ & $8,5 \pm 0,8$ \\
\hline 7 dias & - & - & - & $46,4 \pm \quad 20,4$ & $42,0 \pm 11,9$ & $90,8 \pm 43,3$ & $10,4 \pm 1,1$ \\
\hline 5 Pré-oper. & $12,6 \pm 0,9$ & $6,3 \pm 0,4$ & $41,0 \pm 3,4$ & $63,2 \pm 39,2$ & $30,0 \pm 19,5$ & $146,0 \pm 41,5$ & $8,7 \pm 1,6$ \\
\hline 24 horas & $11,5 \pm 0,9$ & $5,7 \pm 0,3$ & $37,8 \pm 3,4$ & $258,8 \pm 122,8$ & $139,7 \pm 86,9$ & $124,6 \pm 58,2$ & $9,1 \pm 3,1$ \\
\hline 7 dias & - & - & - & $37,0 \pm 19,0$ & $32,2 \pm 6,0$ & $95,0 \pm 9,4$ & $10,8 \pm 1,0$ \\
\hline 6 Pré-oper. & $12,6 \pm 0,7$ & $6,5 \pm 0,5$ & $41,3 \pm 1,6$ & $28,9 \pm 7,0$ & $23,8 \pm 9,9$ & $143,2 \pm 18,4$ & $9,7 \pm 0,7$ \\
\hline 24 horas & $10,8 \pm 1,4$ & $5,6 \pm 0,8$ & $35,2 \pm 3,9$ & $232,8 \pm 115,8$ & $65,9 \pm 25,0$ & $116,5 \pm 20,6$ & $8,2 \pm 1,3$ \\
\hline 7 dias & - & - & - & $52,0 \pm 13,7$ & $22,6 \pm 11,9$ & $97,2 \pm 23,7$ & $9,8 \pm 1,5$ \\
\hline
\end{tabular}

1 = Sem tratamento (controle); 2 = Tratamento com eletrocautério seco; 3 = Tratamento com eletrocautério emplastrado com lidocaína; 4 = Tratamento com eletrocautério emplastrado com neomicina; 5 = Tratamento com eletrocautério emplastrado com glicerina; 6 = Tratamento com eletrocautério emplastrado com vaselina.

A loção de glicerina apresentou bons resultados com relação ao tempo para hemostasia e redução do volume de sangramento. A profundidade das alterações teciduais variou entre 0,6 e 0,8 cm. Entretanto, a intensa fumaça produzida pelo aquecimento dessa substância dificultou a visão do campo operatório. A aspiração constante da fumaça facilita esse o tempo operatório. Entretanto, embolia gasosa por via venosa é relatada em outros trabalhos ${ }^{15}$.
Outra desvantagem dessa substância é a reação inflamatória provocada pela fumaça nos tecidos abdominais e olhos dos cirurgiões, além do fato de ser inflamável a temperaturas superiores a $176^{\circ} \mathrm{C}$.

A pomada de vaselina, ao contrário do gel de lidocaína e da loção de glicerina, não foi boa condutora de corrente elétrica. Essa substância, base para maioria das pomadas, não contém água. Ela atuou como um isolante, 
dificultando a atuação do eletrocautério. A maioria dos animais teve sangramento persistente e o tempo para adquirir a hemostasia e o volume do sangramento foram similares aos do grupo sem tratamento.

As alterações na função hepática são comuns após as manobras de oclusão do fluxo hepático, por causar isquemia tecidual. Neste experimento houve aumento transitório dos níveis séricos de aminotransferases após a ressecção hepática, as quais tiveram um pico com 24 horas e normalizaram até o sétimo dia. Esse padrão de alteração na função hepática é relatado em casos em que outros instrumentos foram utilizados, como o dissector ultra-sônico ou a ablação por radiofrequência, capazes de criar necrose do parênquima hepático, mesmo sem manobra de oclusão do fluxo hepático ${ }^{16,17}$.

Essas alterações da função hepática provavelmente estão relacionadas à necrose das bordas da ferida hepática ${ }^{18}$, e foram proporcionais à profundidade dessa destruição tissular, como mostrou o estudo anatomopatológico.

Em conclusão, dentre as substâncias tópicas testadas neste estudo, o gel de lidocaína e a loção de glicerina foram eficazes na hemostasia do parênquima hepático de coelho.

\section{A B S S T R A C T}

Objective: To assess the hemostasis and healing of the hepatic parenchyma after segmental hepatectomy, using a dry electrocautery or an electrocautery greased with lidocaine gel, neomycin pomade, glycerin lotion, or a vaseline pomade. Methods: Rabbits were submitted to partial hepatectomy and divided into six groups of 10 animals each: Group 1: untreated; Group 2: treated with a dry electrocautery; Group 3: treated with an electrocautery greased with lidocaine gel; Group 4: with neomycin pomade; Group 5: with glycerine lotion; Group 6: with vaseline pomade. Resected liver weight, bleeding volume and time spent to achieve hemostasis were determined. Five rabbits from each group were re-operated upon after 24 hours and five after 7 days in order to obtain a biopsy of the hepatic wound and to explore he abdominal cavity. Red blood cell levels and markers of hepatic function and injury were determined before surgery and before re-operation. Results: Lidocaine gel and glycerine lotion reduced the bleeding volume and the time to achieve hemostasis and conducted the thermal energy of the electrocautery, causing hydropic cell degeneration after 24 hours and deeper necrosis of hepatic tissue after 7 days. All substances increased the aminotransferase concentrations. These values returned to normal after a maximum of seven days. Conclusion: The electrocautery coated with lidocaine gel and glycerine lotion were the most effective methods for the hemostasis of hepatic parenchyma.

Key words: Hepatectomy. Hemostasis, Surgical. Electrocoagulation. Lidocaine. Glycerine.

\section{REFERENCIAS}

1. Nagao N, Goto S, Kawano N, Inoue S, Mizuta T, Morioka Y, Omori $Y$. Hepatic resection for hepatocellular carcinoma: clinical features and long-term prognosis. Ann Surg. 1987; 205:33-40.

2. Gozzeti G, Mazzioti A, Grazi GL, Jovine E, Gallucci A, Gruttadauria S, Frena A, Morganti M, Ercolani G, Masetti M, Pierangeli F. Liver resection without blood transfusion. Br J Surg. 1995; 82:1105-10.

3. Berrevoet F, De Hemptinne B. Use of topical hemostatic agents during liver resection. Dig Surg. 2007; 24:288-93.

4. De Boer MT, Molenaar IQ, Porte RJ. Impact of blood loss on outcome after liver resection. Dig Surg. 2007; 24:259-64.

5. Kooby DA, Stockman J, Ben-Porat L, Gonen M, Jarnagin WR, Dematteo RP, Tuorto S, Wuest D, Blumgart LH, Fong Y. Influence of transfusions on perioperative and long-term outcome in patients folowing hepatic ressection for colorectal metastases. Ann Surg. 2003: 237:860-9.

6. Van Gulik TM, De Graaf W, Dinant S, Busch ORC, Gouma DJ. Vascular occlusion techniques during liver resection. Dig Surg. 2007; 24:274-81

7. Garrison RN, Cryer HM, Howard DA, Polk HCJr. Clarification of risk factors for abdominal operations in patients with hepatic cirrhosis. Ann Surg. 1984; 199:648-55.

8. Lisman T, Leebeek FWG. Hemostatic alterations in liver disease: a review on pathophysiology, clinical consequences, and treatment. Dig Surg. 2007; 24:250-8.

9. Bismuth H, Castaing D, Garden OJ. Major hepatic resection under total vascular exclusion. Ann Surg. 1989; 210:13-9.
10. Ogilvie H. Partial hepatectomy. Br Med J. 1953 2:1136-8.

11. Foster JH, Lundy J. Pathology of liver metastases. Current Probl Surg. 1981;18:157

12. Hayden WH, Gerald DD. Thermal ablation for hepatocellular carcinoma. Gastroenterol. 2004; 127:5167-78.

13. Petroianu A, Cabezas-Andrade MA, Berindoague-Neto R. Operações laparoscópicas conservadoras do baço para tratar dor esplênica por isquemia. Arq Bras Cir Dig. 2007; 20:17-22.

14. Petroianu A, Cabezas-Andrade MA, Berindoague-Neto R. Laparoscopic subtotal splenectomy. Surg Laparosc Endosc Percutan Tech. 2008; 18:94-7.

15. Koo BN, Kil HK, Choi J, Kim JY, Chun DH, Hong YW. Hepatic resection by the Cavitron Ultrasonic Surgical Aspirator increases the incidence and severity of venous air embolism. Anesth Analg. 2005; 101:966-70

16. Ayav A, Bachellier P, Habib NA, Pelicci R, Tierris J, Milicevic M, Jiao LR. Impact of radiofrequency assisted hepatectomy for reduction of transfusion requirements. Am J Surg. 2007; 193:143-8

17. Lau WY, Arnold M, Guo SK, Li AKC. Microwave tissue coagulator in liver ressection for cirrhotic patients. Aust N Z J Surg. 1992; 62:576-81.

18. Scharschmidt BF, Davern II TJ. Biochemical liver tests. In: Seldman M, Scharschmidt BF. editors. Sleisenger \& Fordtran's Gastroenterology and liver disease. Philadelphia: WB Saunders; 2002.p.1112-22. 
Recebido em 05/01/2009

Aceito para publicação em 04/03/2009

Conflito de interesse: nenhum

Fonte de financiamento: nenhuma.

\section{Como citar esse artigo:}

Matos Filho AS, Petroianu A, Alberti LR, Vidigal PVT, Reis DCF, Souza

DM . Hemostasia hepática utilizando eletrocautério seco ou emplas- trado com lidocaína ou neomicina ou glicerina ou vaselina, em coelho. Rev Col Bras Cir. [periódico na Internet] 2009; 36(5). Disponível em URL: http://www.scielo.br/rcbc

\section{Endereço para correspondência:}

Andy Petroianu

E-mail: petroian@medicina.ufmg.br 